variation in the conductivities of these compounds with dilution are given in a plate along with the acid amides.

Nitromethane forms two addition products with the formulae respectively, $\mathrm{CH}_{3} \mathrm{NO}_{2} .2 \mathrm{NH}_{3}$ and $\mathrm{CH}_{3} \mathrm{NO}_{2} \cdot \mathrm{NH}_{3}$. Both compounds lose their ammonia when warmed up to laboratory temperature at the atmospheric pressure.

Tetranitromethane forms one addition product with ammonia having the formula $\mathrm{C}\left(\mathrm{NO}_{2}\right)_{4}, \mathrm{NH}_{3}$. Excess of ammonia above one molecule brings about the decomposition of the tetranitromethane and the formation of the ammonium salt of trinitromethane. This compound is very easily soluble in ammonia and forms a solution possessing an excellent conductivity.

The cyanides of the heavy metals and cyanacetamide, when dissolved in ammonia, show the remarkable phenomenon of a decrease of the molecular conductivity with the dilution in the more concentrated solutions. As the dilution increases the conductivity passes through a minimum and then increases with the dilution in the manner characteristic of salts in general.

The uni-univalent salts show a wide variation in conductivity and degree of ionization with the dilution. Tables and curves are given showing these relations and comparing the behavior of salts in solution in ammonia with their behavior in aqueous solutions.

In conclusion the authors take pleasure in acknowledging their indebtedness to Mr. Alex. Monroe for a liberal grant for carrying on this investigation and to Professor Lucien I. Blake, of the Department of Physics in the University of Kansas, for generously placing at their disposal the facilities of his department.

LELAND STANFORD, JR., UNIYERSITY AND

THE UNIVERSITY OF KANSAS.

[CONTRIBUTIONS FROM THE CHEMTCAL LABORATORY OF HARVARD COLLEGE.]

\title{
A REVISION OF THE ATOMIC WEIGHT OF CADMIUM.
}

Preliminary Paper.-The Analysis of Cadmium Chloride. By Gregory Patl baxter and MUrRay arnold hines.

Received Januaty 5, 1905.

IN a recent determination of the specific gravity of cadmium chloride, ${ }^{1}$ anhydrous cadmium chloride was prepared by ignition

1 Baxter and Hines: Am. Chem.J., 31, 220 (1904). 
of a double chloride of cadmium and ammonium in a current of hydrochloric acid gas, in a state of so great purity that it was considered worth while to make use of the salt for a determination of the atomic weight of cadmium. There is no lack of data concerning the atomic weight of this element, more than twenty different determinations having been made, which gave values between III.77 and II2.39 $(\mathrm{O}=16.000) .^{1}$ Of these determinations those of Morse and Jones ${ }^{2}$ and Morse and Arbuckle ${ }^{3}$ upon the ratio $\mathrm{Cd}: \mathrm{CdO}$, which yielded the value 112.38 , and that of Bucher ${ }^{4}$ upon the ratios $\mathrm{CdCl}_{2}: 2 \mathrm{AgCl}$ and $\mathrm{CdBr}_{2}: 2 \mathrm{AgBr}$ which yielded the values II 2.37 and $:$ r2.39 seem most reliable, and the International Committee on Atomic Weights has adopted the value 12.4 in its reports. Our results indicate that the real value for the constant in question is almost one-tenth of a unit higher than the above value. A critical discussion of the earlier determinations will, however, be postponed until further investigation upon the subject, now under way, is completed.

\section{PURIFICATION OF MATERIALS.}

Cadmium Chloride.-The general method of purification of the cadmium material was that of fractionally precipitating cadmium sulphide. One kilogram of metallic cadmium was dissolved in aqua regia, the solution boiled to expel chlorine and oxides of nitrogen, filtered, and diluted to about 4 liters. The solution contained traces of copper, thallium, nickel, iron and zinc. When a current of hydrogen sulphide was passed through the solution, the first small fraction of cadmium sulphide which was precipitated was dark-colored, nearly black, owing to the presence of copper and thallium. This fraction was removed by filtration and rejected. A second larger fraction of the sulphide, although it contained no appreciable amount of copper or thallium, also was discarded. The third fraction consisted of all that could be precipitated by saturating the solution with hydrogen sulphide. However, this did not contain more than onequarter of the original material, for the solution was very strongly acid, owing to the large excess of acid used in dissolving the metal and the accumulation of the acid formed

2 Clarke : "Constants of Nature, A Recalculation of Atomic Weights." Smithsonian Miscella neous Collections, I897.

2 Am. Chem.J., 14, 26I (1892).

s Ibid., 20, 536 ( 1898 ).

4 Doctoral dissertation, Johns Hopkins University, Baltimore, I895. 
during the precipitation. The solution was separated from the precipitate by decantation, and was then diluted to sixteen liters. Upon saturating this solution with hydrogen sulphide a fourth fraction of cadmium sulphide was obtained, and a second dilution of the solution made possible the precipitation of nearly all the remainder of the cadminm in still a fifth fraction.

The third, fourth and fifth fractions of the sulphide were separately washed until free from chlorides. As the electrolytes were eliminated, the cadmium sulphide showed a tendency to pass into a colloidal condition, which necessitated long standing for the precipitate to settle after each washing, although the flasks which contained the precipitates were kept warm by being placed upon a steam radiator. During the washing the original fine yellow precipitate was gradually converted into an orangered crystalline modification. When, as was usually the case, both forms were present in the same flask, the red form quickly settled to the bottom with a sharp line of division from the yellow form. Nearly all the yellow form was changed into the red modification upon standing about three weeks.

In order to free the sulphide from included and occluded impurities it was dissolved and reprecipitated. The red form of the sulphide was apparently insoluble in dilute sulphuric acid, for in one case the washed sulphide was boiled with the acid for twelve hours without any appreciable amount of solution. Finally, hydrochloric acid was used to dissolve the cadmium sulphide. The solution of each fraction was diluted to eight liters and was saturated with hydrogen sulphide. Since only a portion of the cadmium was precipitated in this way, owing to the large excess of acid, the acid was partially neutralized with ammonia. This resulted in the precipitation of more cadmium sulphide, although the solution still contained considerable cadmium, for cadmium sulphide is soluble to a marked extent in an acid solution of ammonium chloride.

The sulphide obtained from each of the original three fractions both before and after the addition of ammonia was combined and washed until free from chlorides. Each fraction was dissolved in redistilled nitric acid, then enough redistilled sulphuric acid to convert the nitrate into sulphate was added, and the solutions were evaporated and the residues heated until all 
volatile acids were expelled. Finally the sulphate was recrystallized three times from aqueous solution.

The cadmium sulphate was converted into cadmium chloride by first obtaining metallic cadmium electrolytically. A saturated solution of cadmium sulphate was electrolyzed with about one ampere current per square decimeter in a platinum dish, which served as the cathode, until deposition ceased. After the deposit of metal had been thoroughly washed with hot water until free from sulphate, it was dissolved in hydrochloric acid which had been distilled with the use of a platinum condenser.

In order to prepare the double chloride of cadmium and ammonium of the formula $\mathrm{CdCl}_{2} \mathrm{NH}_{4} \mathrm{Cl}$, the calculated amount of ammonium chloride was added to the cadmium chloride and the solution evaporated to crystallization. This ammonium chloride was synthesized from hydrochloric acid and ammonia. The hydrochloric acid had been distilled in platinum, and the ammonia had been freed from amines and purified as follows : Ammonium chloride was boiled with concentrated nitric acid for about twenty hours, and then after crystallization was converted into ammonia by distillation with sodium hydroxide. The solution of pure ammonia was distilled into the pure hydrochloric acid in a platinum dish, and the solution of ammonium chloride was evaporated to crystallization.

The cadmium ammonium chloride was crystallized in a platinum dish, eight times in the case of the first fraction, four times in the case of the second fraction. The third fraction of the sulphide was not converted into the double chloride, but this material will be investigated in a research upon cadmium bromide now in progress. The first fraction is designated as Sample I, Fraction I, the second as Sample I, Fraction 2.

A third specimen used in the analyses was a portion of that employed in the determination of the specific gravity of cadmium chloride. This is designated as Sample 2. The method of purification of Sample 2 was almost exactly identical with that described above, except that the original material was not fractionated with hydrogen sulphide.

Silver.-In the preparation of pure silver essentially the same method was employed as in other atomic weight investigations in this laboratory. The various treatments consisted in thrice precipitating the silver from a strongly acid solution of silver 
nitrate in nitric acid with a large excess of hydrochloric acid, with intermediate reduction of the silver chloride in each case by means of invert sugar and sodium hydroxide. The final product of metallic silver was fused on charcoal in the flame of a blowpipe. Then the buttons were converted into electrolytic crystals, and finally these crystals were fused in a current of electrolytic hydrogen in a boat of pure lime. Richards and Wells, in a recent investigation of the purity of silver purified by different methods, have found that silver prepared in the above fashion is at least as pure as any. ${ }^{1}$ Two different samples, purified in the same way, were employed in this research. One was prepared especially for this investigation and was used in Analyses 4,5 and 6 . The other was a portion of the material employed in an investigation upon the atomic weight of iodine by one of us $^{2}$ (Analyses 7, 8 and 9).

\section{METHOD OF ANALYSIS.}

The method of analysis differed little from that used in the analysis of halogen salts in recent atomic weight investigations in this laboratory. A portion of the cadmium ammonium chloride, contained in a platinum boat in a hard glass tube, was heated in a current of pure dry hydrochloric acid gas until all of the ammonium chloride had been expelled. After the salt had cooled, the hydrochloric acid was displaced by pure dry nitrogen, and this in turn by dry air. The boat was transferred to a weighing-bottle and the stopper inserted without an instant's exposure of the salt to moisture, by means of the bottling apparatus which has been frequently described in papers from this laboratory. ${ }^{3}$ After standing in a desiccator near the balance for some time, the weighing-bottle and boat containing the salt were weighed.

The apparatus used for the expulsion of the ammonium chloride from the double salt was the same as in the work upon the specific gravity of cadmium chloride. ${ }^{4}$ Sublimation of the cadmium chloride always took place to some extent during the heating, and the sublimed salt occasioned some difficulty since it flowed down the inside of the glass tube and, upon solidification,

1 This research is soon to be published by the Carnegie institution.

2 Baxter : Proc. Am, Acad, 40, 4I9 (1904); This Journal, 26, 1577.

3 Richards and Parker : Proc. Am. Acad., 32, 59 (1896).

* Loc cit. 
firmly cemented the boat to the tube. Furthermore, the salt which adhered to the outside of the boat had thus been fused in contact with glass, and hence must have been impure. Both these difficulties were avoided by supporting the boat upon a carriage of heavy platinum wire. While the salt was still warm and the current of hydrochloric acid gas was still passing, the boat was pushed out of the carriage by means of a long glass rod. Neglect to observe the latter precaution usually resulted in the cementing of the boat to the carriage by the salt which had condensed upon the outside of the boat.

It has already been shown that barium and calcium chlorides when they have been fused and allowed to solidify in an atmosphere of hydrochloric acid gas, occlude none of the gas, ${ }^{1}$ for they give neutral solutions; hence it is reasonable to conclude that this is the case with cadmium chloride also. However, in order to test this point, in Analysis 9, the hydrochloric acid was displaced by nitrogen while the salt was still warm, and in Analysis 8 the salt was allowed to solidify only when the hydrochloric acid had been almost completely displaced by nitrogen. In one experiment where the hydrochloric acid had been completely displaced by nitrogen, the boat became covered with a gray coating which turned brown, and finally volatilized when the boat was ignited. This coating undoubtedly consisted of metallic cadmium, formed by the dissociation of cadmium chloride vapor. The close agreement of the results of Analyses 8 and 9 with those obtained in the other analyses where the salt solidified and cooled in hydrochloric acid, shows conclusively that no appreciable amount of hydrochloric acid was occluded by the salt.

It was shown in our determination of the specific gravity of cadmium chloride, that the salt when prepared in this way contains no ammonium chloride. It is probable that the cadmium chloride contains no basic compound, since no insoluble salt is produced when the chloride is dissolved in water. The aqueous solution of the salt invariably contained a few tenths of a milligram of black insoluble matter which consisted chiefly of platinum. The presence of this platinum was undoubtedly due partially to slight attacking of the boat, owing perhaps to contamination of the hydrochloric acid with traces of air. The slight

1 Richards : Proc. Am. Acad., 29, 59 (1893); This Journal, 24, 376 (1902). 
loss in weight of the boat which resulted in most of the analyses was not sufficient to account for all the insoluble residue, which, therefore, must have had its source, in part, in the original material. Whether the platinum was dissolved from the platinum condenser during the distillation of the hydrochloric acid, or from the platinum dish during the solution of the cadmium is uncertain. At all events, the temperature to which the salt was heated must have been sufficient to decompose all the platinic or platinous chlorides present, and since the insoluble residue was filtered out and weighed, and corrections applied to the weight of the salt both for the loss in weight of the boat and for the insoluble matter, no appreciable error could have been introduced by the platinum.

After the salt had been weighed, the boat was transferred to a flask and the salt was dissolved in about $200 \mathrm{cc}$. of the purest water. The weighing-bottle was rinsed and the rinsings were added to the solution. Next the solution was filtered into the precipitating flask through a tiny filter to collect the insoluble matter. Filter-paper and residue were then ignited in a weighed porcelain crucible.

In the preliminary analyses the ratio of cadmium chloride to silver chloride was determined by adding to the solution of cadmitum chloride, which had been diluted in the precipitating flask until not stronger than $\mathrm{I}$ per cent., a solution of a slight excess of silver nitrate of very nearly the same concentration. The flask, which was provided with a ground-glass stopper, was shaken for some time, and was allowed to stand until the sulution was clear. Then the precipitate of silver chloride was transferred to a Gooch crucible, after it had been washed by decantation six to eight times with about $550 \mathrm{cc}$. of a very dilute silver nitrate solution, and finally several times with pure water. Needless to say, the operations of precipitation and filtration were performed in a room lighted only with ruby light. The crucible with the precipitate was placed in an air-bath and heated for several hours at $130^{\circ}-140^{\circ} \mathrm{C}$, and after it had cooled in a desiccator it was weighed. In order to determine how much moisture was retained by the precipitate in each case, it was transferred to a clean porcelain crucible and weighed, then the salt was fused by heating the small crucible, contained in a larger covered crucible, and again weighed. Two different specimens 
of silver chloride from analyses were separately dissolved in ammonia and reprecipitated with hydrochloric acid, and the filtrate, after evaporation, was tested for cadmium. Negative results were obtained in both cases.

The determination of the silver chloride dissolved in the washwaters was the most difficult step in the analysis. At first the last few washings, those which had been carried out with pure water and which were the only ones which could have contained dissolved silver chloride, were evaporated to small bulk and an excess of silver nitrate was added. The precipitate of silver chloride, together with any asbestos which had been displaced from the Gooch crucible were collected upon a small filter which was ignited and weighed. Owing to the combined effect of organic matter and light upon these solutions the precipitate was always too heavy. Hence this method was finally discarded. Four preliminary results obtained in this way varied between I 2.393 and I 2.455 for the atomic weight of cadmium.

In order to avoid this error, in the final series the silver chloride dissolved in the wash-waters was determined by precipitating the chloride in 25 -cc. portions of the solution with an excess of silver nitrate and comparing in a nephelometer ${ }^{1}$ the precipitate produced with that from solutions prepared from standard hydrochloric acid solutions. At least two comparisons were made in each analysis.

The weight of pure silver required exactly to combine with the chlorine in cadmium chloride was also determined. From the weight of cadmium chloride very nearly the necessary quantity of pure silver was calculated. This silver was weighed out and dissolved, in a flask provided with a column of bulbs to prevent loss of silver by spattering, in distilled nitric acid diluted with an equal volume of water. After the silver was dissolved, the solution was diluted somewhat and heated until free from nitrous fumes. Then it was diluted until not stronger than i per cent., and was slowly added to the I per cent. solution of cadmium chloride in the precipitating flask. After several minutes' shaking it was allowed to stand several days, with occasional shaking, until the solution was perfectly clear. Portions of the solution were then tested in the nephelometer for excess of chlorine or silver, and, if necessary, either standard silver nitrate or hy-

1 Richatds and Wells: Am. Chem.J., 31, 235 (I904). 


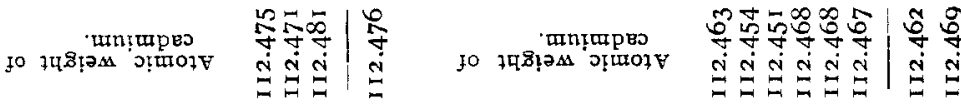

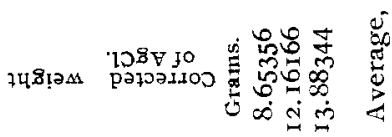

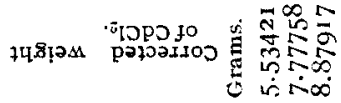

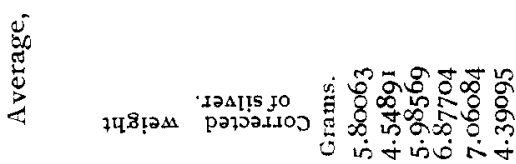
造 要要 赵

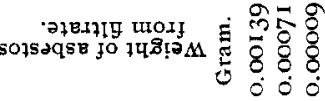

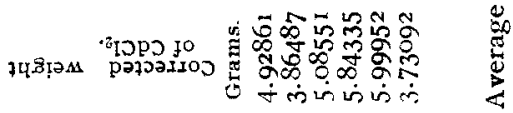

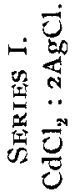

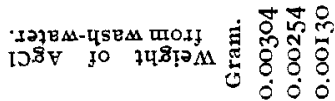

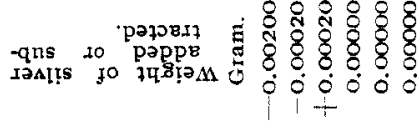

mnnoes क⿻

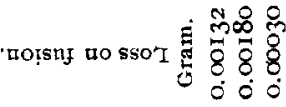

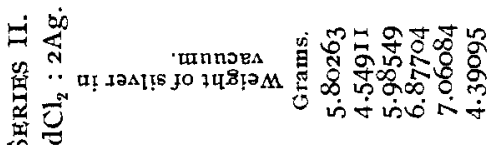

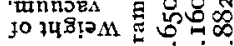

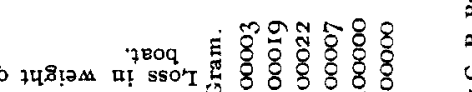

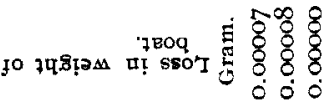
jo 
drochloric acid solution was added, and the process of shaking and testing repeated until the amounts of chlorine and silver in the solution were equivalent.

A vacuum correction of $+0.00015^{6}$ gram was applied for every apparent gram of cadmium chloride, of +0.000075 gram for every apparent gram of silver chloride, and of $-0.00003 \mathrm{I}$ gram for every apparent gram of silver.

All weighings were made by substitution, with tare vessels as nearly as possible like those weighed. The gold-plated brass weights were twice carefully standardized to hundredths of a milligram.

In the calculations the atomic weight of silver is assumed to be 107.93 , and that of chlorine 35.473 , according to the very recent work of Richards and Wells.

The close agreement of the results in each series leaves little doubt of the identity of the different samples, although they represent material from different sources as well as different fractions of the same material. The slight discrepancy between the results by the two methods is undoubtedly due, in part, to the difficulty in determining silver chloride with accuracy, owing, in the first place, to loss of chlorine by the silver chloride in the processes of manipulation and drying, and in the second place to the slight solubility of silver chloride even in dilute silver nitrate solutions. In the light of these possibilities, it is probable that the results of Series I are slightly too high. On the other hand, it is probable that the average of Series II is slightly too low, for the average of Experiments 7, 8 and 9, in which the experience gained in the previous analyses was a very considerable aid, is I12.468, 0.006 of a unit higher than the average of the whole series. The average of Series I and II, I 2.47, undoubtedly represents very closely the true atomic weight of cadmium ( $\mathrm{Ag}=107.93)$. If the atomic weight of silver referred to oxygen ( 16.000 ) is assumed to be 107.92 , as seems probable from Richards and Wells' work upon chlorine, the atomic weight of cadmium is lowered to I 2.46 .

The atomic weight of cadmium is still under investigation in this laboratory, through the analysis of cadmium bromide.

We are indebted to Dr. Wolcott Gibbs and to the Cyrus M. Warren Fund for Research in Harvard University for many indispensable platinum vessels.

CAMBridge, MAss., December 3I, Ig04. 